\title{
Retos y experiencias en la construcción de un peritaje antropológico con perspectiva de género: la lucha de Bertha por la tierra en Chiapas
}

\author{
LAURA EDITH SAAVEDRA HERNÁNDEZ
}

\begin{abstract}
En este artículo se narra la experiencia de la construcción de un peritaje antropológico con perspectiva de género y los retos que implica trabajar con ésta en las costumbres de las comunidades indígenas en México. El Centro de Derechos de la Mujer de Chiapas, A. C., Ileva la defensa del caso y aplica la metodología de defensa participativa, cuyo objetivo es empoderar a las mujeres indígenas en la defensa de sus derechos. El peritaje procura dar testimonio de las permanencias y continuidades en las costumbres indígenas y las desigualdades de poder y género cuando las mujeres indígenas buscan justicia.
\end{abstract}

PALABRAS CLAVE: peritaje antropológico, perspectiva de género, despojo de tierras, derechos de las mujeres, mujeres indígenas

Challenges and Experiences in the Construction of an Anthropological Expert Witness Report with Gender Perspective: Bertha's Struggle for Land in Chiapas

This article discusses the experience of the construction of an anthropological expert witness report with a gender perspective and the challenges of working with this perspective in the customs of the indigenous communities in Mexico. The defense of the case is carried through the Center of Rights the Women of Chiapas, who applied the methodology of participatory legal defense, which seeks to empower indigenous women in the defense of their rights. The anthropological expert witness report aim to describe the permanence and continuity of the indigenous customs and inequalities of power and gender when indigenous women seek justice.

Laura Edith SaAvedra Hernández

Centro de Investigaciones y Estudios Superiores en Antropología SocialCiudad de México, México lauedith29@hotmail.com
KEYWORDS: anthropological expertise, perspective of dispossession of lands, gender, indigenous women, women's rights 


\section{Introducción}

1 propósito de este artículo es mostrar la experiencia de intercambio colaborativo con el Centro de Derechos de la Mujer de Chiapas, A. C. (CDMCH), mediante la construcción de un peritaje cultural que me fue encargado debido a la aproximación al campo de estudio de mi investigación para tesis doctoral, que tuvo como uno de sus objetivos elaborar una metodología colaborativa con el $\mathrm{CDMCH}$ (Saavedra, 2018). El peritaje formó parte de la defensa de un caso jurídico en el que una mujer indígena tseltal de la comunidad La Grandeza, del municipio de Amatenango del Valle, fue acusada de despojo de tierras. El caso de Bertha ejemplifica los retos y la experiencia propia y de las abogadas del CDMCH en la elaboración de un peritaje con perspectiva de género.

Con la presentación de este informe pericial se intentó exponer las desigualdades que prevalecen en las comunidades indígenas respecto al acceso de las mujeres a la tierra en tenencia, uso y usufructo. Construir un documento con estas características no fue fácil, pues en la actualidad existen pocos peritajes en México que retomen la perspectiva de género como centro de su análisis en casos de acceso a la tierra en comunidades indígenas. ${ }^{1}$ Fue complicado tener precedentes escritos y probados que nos dieran luz para alcanzar nuestro objetivo, y al mismo tiempo, era una oportunidad para sentar las bases de futuras investigaciones de esta naturaleza.

Para esta investigación me he colocado como feminista comprometida con la lucha contra las violencias hacia las mujeres. Por medio de esta propuesta pretendo

$1 \quad$ Aunque en otros países existen peritajes con perspectiva de género en el análisis jurídico, para éste sólo consultamos el elaborado por Juan Carlos Martínez y Daniela Ramírez (2012), cuyo objetivo era identificar los daños individuales, familiares y comunitarios ocasionados por la cesárea deficiente que se le practicó a Irene Cruz Zúñiga y determinar las medidas de reparación para las víctimas directas e indirectas de acuerdo con el contexto cultural y la perspectiva de género. El documento fue proporcionado por los peritos. 
dar a conocer de una manera respetuosa las situaciones de violencia que viven las mujeres en su encuentro con las justicias. Mi compromiso investigativo es no revictimizar a las personas que han querido compartir su vida conmigo.

Mi postura coincide con Haraway (1995), acerca de que situar el conocimiento nos brinda la oportunidad de cuestionar los criterios de verdad construidos alrededor de los conceptos. En la investigación del peritaje cultural doy testimonio de que mi análisis estuvo cruzado por mi experiencia, mi militancia, mi género, mi identidad mestiza y mi clase, lo que con seguridad me dio o quitó privilegios epistémicos sobre ciertas discusiones. Situar mi conocimiento también me ayudó a afirmar que lo que analizo o digo conlleva toda la ética y el respeto posibles hacia la mujer tseltal que compartió aspectos de su vida; el CDMCH, que me abrió sus puertas para hacer la investigación más extensa y conmigo misma y mi ética profesional, como mujer y feminista.

Con base en lo anterior, este artículo aporta al conocimiento empírico de los retos que supone la elaboración de un peritaje cultural con perspectiva de género y plantea algunas discusiones actuales sobre peritajes culturales. Por otro lado, también intento abonar a los ejemplos empíricos sobre el tratamiento de las metodologías colaborativas, lo que constituye una contribución a la vida de las mujeres que deciden participar en nuestras investigaciones. Señalo que la investigación comprometida también es una forma de activismo y un punto de partida para construir investigaciones más éticas y comprometidas con las realidades sociales.

\section{Retos en la construcción de un peritaje cultural o antropológico con perspectiva de género}

Sin duda, la aprobación del Convenio 169 (CDI, 2003) de la Organización Internacional del Trabajo $(\mathrm{OIT})^{2}$ permitió que se tomara en cuenta la pluralidad cultural en la legislación mexicana. Este hecho fue una importante oportunidad para el reconocimiento de las formas de vida de los pueblos indígenas y los sistemas normativos en los que se basa su cultura. En México, la reforma al Código Federal de Procedimientos Penales del 8 de enero de 1991 (Congreso de la Unión, 1991) trajo consigo la obligación de los jueces de contar con peritajes antropológicos y culturales, así como de proporcionar traducción obligatoria en los casos en que estuvieran involucradas personas indígenas. Esta reforma implicó nuevos retos para el sistema de justicia en la inclusión de las culturas indígenas y abrió la posibilidad de reconocerlas como sujetos de derechos. Las variaciones a la Ley Penal de 1991 fueron significativas porque dejaban atrás el reconocimiento aislado de las "costumbres" proyectadas por un individuo y valoraban la forma colectiva de las comunidades para resolver sus conflictos (Escalante, 2002).

Sin embargo, 26 años después, las personas indígenas que se enfrentan al sistema penal o a la procuración de justicia siguen viviendo discriminación y exclusión. En la experiencia que analizaré en este artículo constaté que la intención de incluir los derechos de la población indígena en las leyes sigue siendo un camino en construcción. En mi estancia de un año en los Altos de Chiapas observé que los servicios jurídicos estatales siguen teniendo deficiencias respecto a la atención de casos que involucran personas indígenas, por ejemplo, en los juzgados civiles y penales de la región hay pocos traductores certificados, los cuales se reparten el trabajo en los distritos judiciales. Esto implica una espera de más de dos horas en el juzgado para que se atienda un caso o se haga una declaración en una lengua diferente al español. Los obstáculos que enfrentan los

2

En 1989, durante la 76 Reunión de la Conferencia General de la oır. Entró en vigor en México en 1990, con su publicación en el Diario Oficial de la Federación. 
pueblos indígenas para acceder a la justicia siguen siendo una realidad en México.

A pesar de que este contexto permea la mayoría de las prácticas jurídicas, gracias a las luchas de organizaciones civiles indígenas y no indígenas que han puesto en evidencia estas deficiencias, el Estado ha hecho un esfuerzo por reconocer las diferencias $\mathrm{y}$ tender puentes de intercambio y entendimiento entre las culturas indígenas y el derecho estatal, para respetar los derechos de éstas en la medida de lo posible. En este sentido, las posibilidades de los peritajes antropológicos para lograr este reconocimiento son infinitas, como menciona Valladares: "la estrategia de poder que el peritaje contrapone es, metodológicamente, la posibilidad de cuestionar, con otro saber, ese conocimiento hegemónico que busca imponerse, a través de desconocer o por despreciar y subvalorar al otro diferente y no hegemónico" (2012: 12).

Construir un peritaje antropológico es un reto importante para el antropólogo, pues conlleva mucha responsabilidad y un gran sentido de la ética para con las prácticas que se analizan. En palabras de Sánchez, se intenta construir un "rompecabezas para describir las costumbres de otros pueblos, de manera que sean plausibles para las y los juzgadores y les lleguen como aportes para sus determinaciones" (2010: 341).

Saliendo de la concepción de que los peritajes culturales o antropológicos sólo deben fungir como una especie de "traducción cultural" para los juzgadores, en este artículo, la construcción del peritaje no sólo se enfocó en...

reducir [la cultura] a los términos del derecho positivo ni transcribir sus normas orales al lenguaje escrito, sino [en] exigir un espacio en el sistema jurídico hegemónico para la pluralidad, para la diversidad, para la diferencia. Un espacio que permita a los pueblos indígenas contar con una vía propia de desarrollo y expresar sus intereses en un plano de igualdad con el Estado, pero también para que el resto de los ciudadanos nos encontremos con nuestro derecho a la participación social y política plenamente reconocida, no sólo en el nivel electoral sino en toda la pluralidad, la diversidad y la diferencia que permita la conformación de un Estado plural (Ortiz, 2000: 80).

Para poder dar testimonio de la pluralidad, los tiempos actuales han exigido al sistema jurídico mexicano que no sólo tome en cuenta la diferenciación étnica, pues como lo plantea la aproximación de la interseccionalidad, ${ }^{3}$ las opresiones y las violencias que se viven en las realidades sociales son intersectadas por otras categorías que construyen las desigualdades, como el género y la clase. De esta manera, cuando confluyen las posiciones de los sujetos en las sociedades, las opresiones se profundizan al imbricarse estas diferencias.

De ahí que en las últimas décadas, con el aumento de la denuncia de violencia contra las mujeres, las organizaciones de la sociedad civil impulsen una conciencia de la necesidad de que, en los casos jurídicos que involucran violencias de género, los jueces no sólo se alleguen peritajes culturales que ayuden a dar un sentido a las formas de vida de los pueblos indígenas, sino que también se construyan con perspectiva de género para exponer que las violencias que viven las mujeres son producto de un sistema de desigualdades de poder entre hombres

La tendencia a tratar género y etnia como categorías mutuamente excluyentes produce consecuencias problemáticas tanto en la teoría feminista como en las políticas antirracistas. En concreto, Crenshaw (1995) entiende que al limitar el examen de las discriminaciones a las experiencias de los miembros más privilegiados de las minorías -los hombres negros y las mujeres blancas-, se margina del análisis a aquellos que sufren múltiples barreras, que no pueden ser comprendidas como consecuencia de rasgos de discriminación aislados, por lo tanto, las mujeres negras son "invisibilizadas". 
y mujeres, fincadas en un sistema patriarcal que las victimiza. Esto ha ayudado no sólo a visibilizar el origen de las violencias basadas en el género sino también a que los juzgadores tengan en cuenta el contexto en el que se desenvuelven dichas violencias y construyan juicios que promuevan la igualdad y la no violencia contra las mujeres.

Estos derechos se encuentran plasmados en la Convención Americana sobre Derechos Humanos (Presidencia de la República, 1981), la Convención Interamericana para Prevenir, Sancionar y Erradicar la Violencia contra la Mujer o "Convención de Belém do Pará" (SRE, 2008) y en la Ley General de Acceso de las Mujeres a una Vida Libre de Violencia, que fue aprobada en 2007 (Congreso de la Unión, 2007). ${ }^{4}$ Cada una de estas legislaciones marca las obligaciones del Estado mexicano para que las mujeres tengan acceso a la justicia desde la igualdad de género. En ese sentido:

Realizar un peritaje con perspectiva de género implica proveer una explicación de hechos o circunstancias que toman en cuenta las relaciones desiguales de género, las relaciones de poder y la situación de discriminación en las que se encontraba la víctima por su situación y condición de género. El objetivo del peritaje será identificar y visibilizar hechos y/o circunstancias que se han naturalizado y se consideran "normales" y así entender cómo opera la discriminación contra las mujeres y visibilizar las razones de género que llevaron a la muerte [o a la violencia] (Estrada, 2016: 22).

Elaborar peritajes interdisciplinarios ayuda a cuestionar las "costumbres y tradiciones" de las comunidades indígenas, que por años han excluido a las mujeres de la participación comunitaria, y las leyes y discursos de género del Estado, que también han contribuido a construir representaciones de "retraso cultural", que plantean que los pueblos indígenas son violentos "por naturaleza". La interdisciplinariedad en la construcción de este peritaje nos comprometió, como se verá, a ir más allá de sólo "presentar una prueba" sobre la legitimidad de la herencia de Bertha y el funcionamiento de este aspecto en las formas de vida de la comunidad. Pensamos que sólo describirlo excluía la posibilidad de visibilizar que estas "costumbres" de la herencia de la tierra están supeditadas a cambios ligados a contextos más amplios.

La inclusión de la perspectiva de género daba la oportunidad de desesencializar las prácticas comunitarias y sociales y exponer que la forma de vida y la realidad de las mujeres en la comunidad también están ligadas a contextos sociales, políticos y económicos más amplios que han afectado la vida en las comunidades, y que las violencias contra las mujeres se relacionan con estos contextos. Ésta era una posibilidad de desestabilizar los discursos culturales y de género hegemónicos que han excluido a los pueblos indígenas y a las mujeres de estas comunidades, que no están dentro de las concepciones hegemónicas de la sociedad.

Incluir la perspectiva de género no fue tarea fácil. Por un lado, se tenían que presentar pruebas que hicieran patentes las formas de vida de la comunidad de La Grandeza respecto a las “costumbres” de la herencia, diferentes de las formas de herencia descritas en las leyes positivas; por otro lado, sin deslegitimar esa forma de vida, era necesario mostrar las contradicciones que pueden tener con los derechos de las mujeres. Se discutían las tensiones entre el derecho colectivo a la autodeterminación de los pueblos y los derechos individuales de las mujeres a la propiedad, a la toma de decisiones y a no vivir violencias.

4

Existen otros instrumentos jurídicos, internacionales y nacionales, que clarifican los derechos de las mujeres; sin embargo, los que he mencionado hacen especificaciones importantes sobre el acceso a la justicia. 
Para salir de esta encrucijada tuvimos que llevar a cabo un análisis contextualizado, lo que nos permitió ilustrar cómo algunas "costumbres" violen$\tan$ a las mujeres y conocer que no todas las formas de vida tradicionales de las comunidades permiten las violencias.

Fundamentar y contextualizar que las formas de vida comunitaria y las violencias contra las mujeres son parte de contextos específicos puso de manifiesto que, si bien existían "costumbres" que violentaban a las mujeres y cooptaban sus derechos, también existían resistencias y cuestionamientos en las mismas "costumbres", en las que las mujeres, lejos de ser personas pasivas que sólo reciben la violencia, han buscado formas de enfrentar y dialogar con sus autoridades para luchar juntos contra lo que les hace daño.

Estos hallazgos nos hicieron ver que la construcción de peritajes culturales o antropológicos con perspectiva de género tiene implicaciones éticas importantes. Afirmar o deslegitimar "costumbres" u opresiones impacta las dinámicas de las comunidades y la vida de las mujeres. Nos dimos cuenta de la importancia de construir conocimientos situados que no violenten ni revictimicen a las mujeres y a los pueblos indígenas.

A continuación, se mostrarán las experiencias de quien suscribe y de las abogadas del CDMCH en la elaboración de un peritaje antropológico con perspectiva de género y cómo éste no sólo se convirtió en una prueba jurídica, sino en una investigación que sostiene que la defensa jurídica que lleva a cabo el CDMCH abarca los derechos humanos y de las mujeres.

\section{El caso de Bertha y la lucha por el derecho a la tierra}

En septiembre de 2014 me encontraba en la ciudad de San Cristóbal de Las Casas, Chiapas, haciendo el acercamiento al campo para la elaboración de mi tesis de doctorado. Propuse un esquema de trabajo colaborativo, pues me interesaba conocer cómo el CDMCH aplicaba su metodología de defensa participativa en casos jurídicos de mujeres indígenas que viven violencias. Después de participar un tiempo como voluntaria y familiarizarme con los casos, pude colaborar en la construcción de un peritaje antropológico con perspectiva de género para demostrar la exclusión que vivía una mujer indígena acusada de despojo de su solar y su tierra.

Bertha es una mujer tseltal de 41 años de edad, originaria de un municipio de los Altos de Chiapas. Estuvo casada con Francisco durante 13 años y tuvo con él tres hijas y un hijo. Durante su matrimonio estuvo expuesta a la violencia física y psicológica, pero la aguantó, "como una mujer de su comunidad lo haría”. Sin embargo, después de cada episodio de

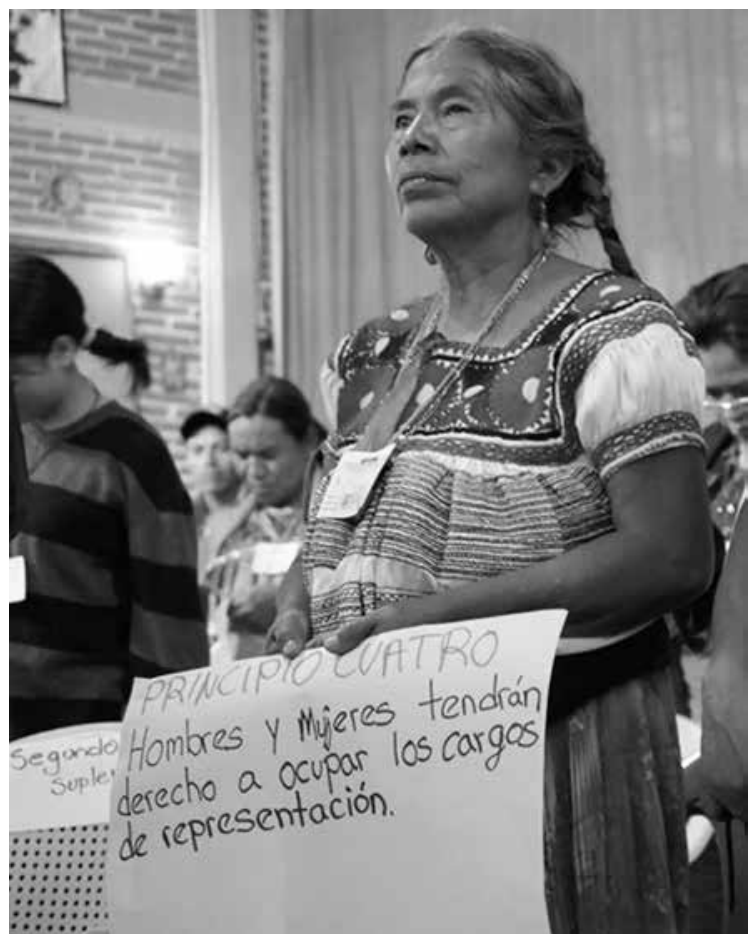

Laura Edith SaAvedra Hernández • Asamblea del Movimiento en Defensa de la Tierra y el Territorio y por la Participación de las Mujeres en la Toma de Decisiones, San Cristóbal de Las Casas, Chiapas, marzo de 2017. 
violencia, como es la costumbre de su comunidad, Bertha mandaba llamar a su mamá, sus suegros, su padrino y madrina de bodas para que hablaran con Francisco y le dijeran que lo que hacía no estaba bien. Cada vez él prometía que ya no la insultaría ni la trataría mal, que eso no se repetiría. Así pasaron 13 años.

Por las violencias que vivía, Bertha se apartó de algunas actividades, como las reuniones de mujeres indígenas de la Coordinadora Diocesana de Mujeres (Codimuj) y los encuentros con sus amigas y visitas a su mamá, entre otras cosas. Se dedicaba por completo a sus hijos y a criar pollos, que vendía periódicamente en la ciudad de San Cristóbal de Las Casas. Aunque dejó de hacer las cosas que alegraban su corazón, Francisco la trataba mal y la violentaba.

Bertha decidió dejar a Francisco el día en que él le pegó en la cara y le dejó un ojo morado y un brazo lastimado. Bertha cuenta que, después de ese día, estaban en una reunión familiar y unos invitados comentaron que su hija mayor ya estaba creciendo y pronto la haría suegra. Ella, jugando, dijo que esperaba que su hija consiguiera un buen esposo, que le diera una buena vida. Francisco, muy enojado, interrumpió la conversación. Al llegar a su casa golpeó a Bertha y en la trifulca también lastimó a su hija menor.

Bertha salió de su casa y se fue con su vecina. Francisco le dijo que no regresara nunca, que no tenía nada que hacer ahí. Al día siguiente, acompañada de su mamá y su cuñada, Bertha fue a ver a los jueces de paz y conciliación indígena de la comunidad, quienes se encargan de arreglar los problemas de violencia entre las parejas y familiares, entre otros pleitos que puedan llegar a suceder en las comunidades indígenas del municipio de Amatenango del Valle.

Los jueces de paz, miembros de la comunidad, recomendaron a Bertha que fuera con la procuradora del Sistema Nacional para el Desarrollo Integral de la Familia (DIF), pues ahí se atienden casos de violencia contra menores de edad, mujeres y adultos mayores, principalmente, que pasan a las instancias jurídicas del estado. Ya en la Procuraduría, la encargada revisó las heridas de Bertha, tomó fotos de sus lesiones y su declaración. Mientras esto sucedía, una persona avisó a Francisco que los policías iban por él y que lo más seguro era que lo metieran a la cárcel municipal. Él se fue de su casa y se escondió por unos días.

Después de estos acontecimientos y de que Francisco apareciera, la procuradora citó a ambas partes. Les comentó que lo mejor era conciliar el problema. Bertha le dijo que no quería volver con su esposo, que quería divorciarse y una pensión alimenticia. La procuradora aseguró que entendía a Bertha y expuso que tratar el caso así significaba perder mucho tiempo, pues implicaba ir al ministerio público y levantar una demanda, lo que también significaba gastar dinero; por eso prefería conciliarlo en su oficina, pues les convendría a los dos. La procuradora dejó que Bertha y Francisco hablaran a solas para que arreglaran su problema y llegaran a un acuerdo, en tanto que ella escribiría un acta para comprometerlos a cumplirlo.

En su testimonio, Bertha mencionó que entró con Francisco a una oficina y hablaron. Ella estaba convencida de no regresar con él, así que no hablaron de ese tema, sólo de cómo quedarían en la separación. Francisco mencionó que Bertha no podía quedarse en la casa en la que vivían porque era de su papá y él había dicho que si ella no vivía con él tenía que irse. Bertha le dijo que estaba bien, siempre y cuando le hiciera una casa igual, porque cuando su papá les regaló esa casa había sólo un cuarto y durante 13 años ella también había aportado trabajo y tiempo para construir otro cuarto, el baño, las galeras de los pollos, sembrar árboles frutales, matas de café, etc. Si iba a salirse, quería una casa igual para vivir con sus hijos. Francisco aceptó. Bertha también le pidió una pensión para sobrevivir y él accedió. 


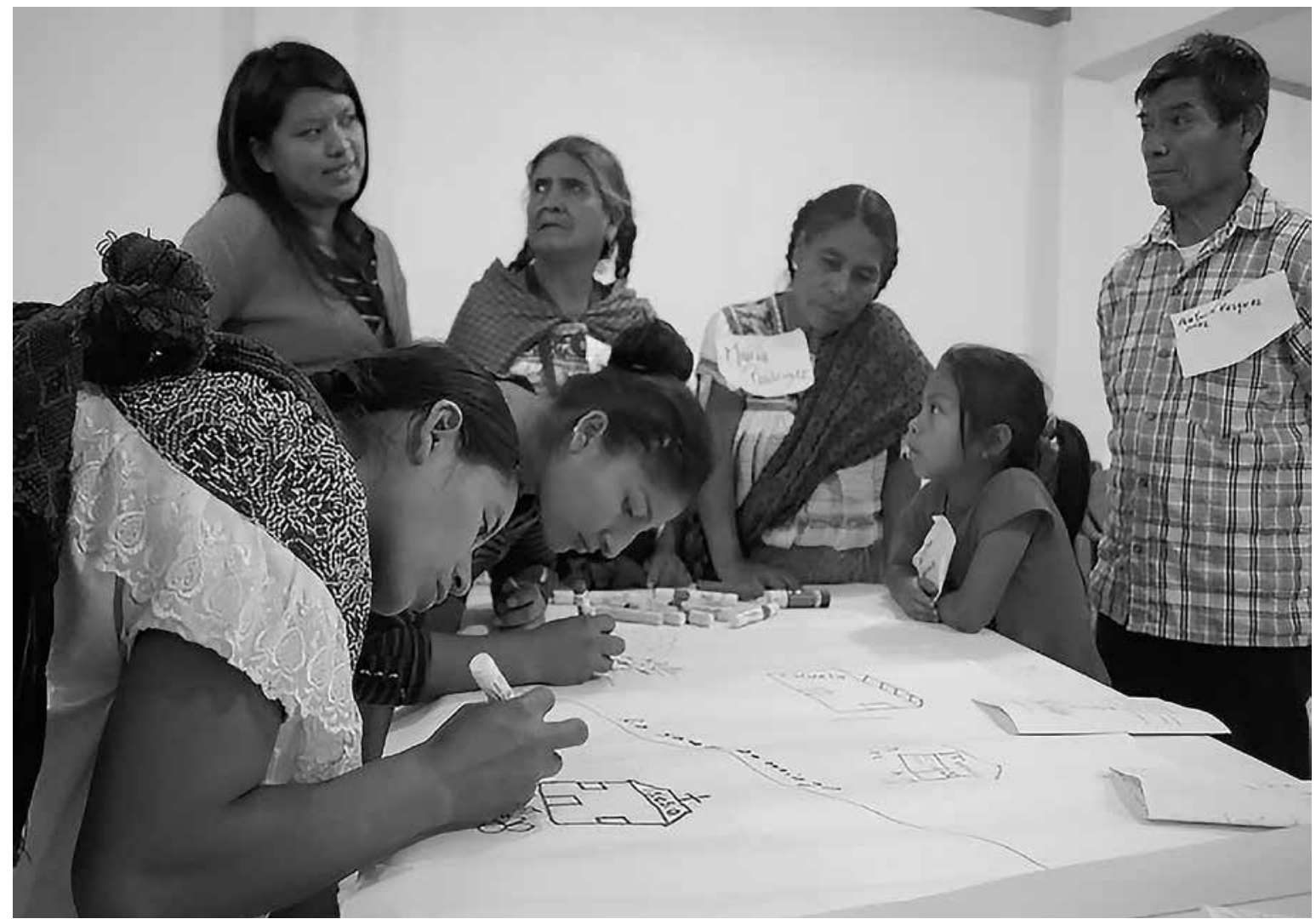

Laura Edith SaAvedra HernándeZ • Mujeres y hombres Kasesel K'op, quienes llevan y traen la palabra, San Cristóbal de Las Casas, Chiapas, junio de 2016.

Al salir, mencionaron los acuerdos, y los presentes, incluyendo a su suegro, quien reclamaba la casa, firmaron el acta. Sin embargo, Bertha, sintió que el trato de la casa era injusto, pues tenía 13 años viviendo ahí y había invertido tiempo y dinero en su cuidado. Además, había sido un regalo de bodas de su suegro, en forma de herencia. Si se apegaba al hecho original, ella tenía el derecho legítimo de quedarse con la casa. Aunque ya había firmado el acuerdo, Bertha comenzó a informarse sobre sus derechos. Así fue como el caso llegó al CDMCH.

Se expuso primero el problema de la propiedad porque el Centro da atención jurídica a varios tipos de violencias contra las mujeres —indígenas y no indígenas-y uno de sus ejes principales es evidenciar el despojo de la tierra contra mujeres indígenas.
La mayoría de sus casos judiciales se centra en la defensa de la tierra o la herencia para las mujeres. Por esta razón, Bertha llegó al Centro a exponer el caso de la propiedad de su casa y no de las violencias vividas. Además, pensó que los problemas de la separación y la pensión se habían resuelto con la conciliación hecha en el DIF.

Al retomar un caso judicial, lo primero que hace el $\mathrm{CDMCH}$ es una investigación a profundidad de los casos y las personas involucradas. Con la ayuda de una promotora de derechos, una mujer tseltal con un amplio conocimiento de las costumbres en las comunidades indígenas de los Altos de Chiapas, se decidió, como parte de la metodología, hacer un mapeo de las autoridades locales encargadas de conciliar o arreglar el caso de la tierra y la 
propiedad, revisar el acta de acuerdos y entrevistar a profundidad a Bertha y a una de sus cuñadas, testigo del maltrato de Francisco. También se hizo una reunión en psicología para ver cómo estaban los hijos después de las violencias vividas. Se hicieron entrevistas y grupos focales con actores de la comunidad, que nos explicaron cómo funcionaba ahí el derecho a la tierra de las mujeres. Por último, se hicieron algunas reuniones de "análisis de la realidad", con el área de formación del Centro, para tener más claro el problema y determinar cuál sería la mejor estrategia para apoyar a Bertha. Es importante mencionar que, como parte de la metodología de defensa participativa, ella se involucró en la elaboración de la estrategia para su defensa jurídica.

Se definió que lo primero era ir a una audiencia con el comisariado ejidal, para conocer las posibilidades reales de Bertha para quedarse a vivir en su casa. Se investigó que en la comunidad de La Grandeza, en casos de violencia con golpes fuertes y sin arreglo para seguir viviendo juntos, por lo general las esposas se quedan en la casa del esposo, más si hay hijos de por medio, lo que era un precedente para la defensa (grupo focal, Colectivo de Mujeres de Amatenango del Valle, La Grandeza, Chiapas, marzo de 2015).

En la consulta con el comisariado ejidal se mencionó que, por el tiempo que había vivido ahí, Bertha podía reclamar la casa como suya y buscar un juicio agrario para tener escrituras de la propiedad. Se formuló la opción de citar a los cónyuges para llegar a una conciliación. Bertha accedió a hablar en el juzgado local con el comisariado ejidal para ver la posibilidad de llegar a un arreglo, sin tener que acudir a la justicia estatal, pues eso lleva más tiempo y más recursos económicos.

El encuentro para la conciliación se llevó a cabo un domingo por la mañana. En la mesa de la comisaría municipal había siete hombres representantes de las comunidades o barrios, y al centro estaba el comisariado ejidal. Nos sentamos en las bancas frente a ellos. De un lado estaban los hermanos de Bertha, su mamá y las integrantes del CDMCH; del otro lado, la cuñada, su esposo, el suegro y el abogado. Sentaron a Bertha y Francisco hasta adelante. El comisariado ejidal dijo por qué estábamos ahí. Mencionó que por esta vez dejarían que los abogados estuvieran presentes en la audiencia, pero que eso no era parte de su costumbre. Se le dio la palabra a Bertha, quien prefirió hablar en tseltal. Comenzó a narrar que el motivo de su separación era que Francisco la golpeaba y contó su historia, que ella y sus hijos vivían en la casa que su suegro les había regalado a ella y a su esposo el día de su boda, que era un pequeño cuarto, y con el tiempo habían trabajado para ampliarla y sembrar árboles. Dijo que se acercaba a ellos como autoridades porque sabía que cuando había un conflicto de violencia en la comunidad y los esposos se separaban, las mujeres podían quedarse en sus casas con sus hijos, por lo que no entendía por qué le querían quitar su casa.

El suegro levantó la mano y le dijo en tseltal: "entiendo que yo te di esta casa cuando te casaste con mi hijo, en la ceremonia del civil te la di, pero ahora que ya no vives con mi hijo no te la puedo dejar, porque si encuentras otro esposo, él se quedará con mi tierra". ${ }^{5}$ Bertha le dijo que no quería tener otro esposo y que necesitaba la casa por sus hijos, que eran sus nietos, y que si no vivía ahí iban a sufrir y a no tener donde dormir, que se venía el invierno y no entendía por qué querían sacarla de ahí con esos argumentos.

El comisariado preguntó al suegro de Bertha: “¿usted tiene un papel que diga que es suyo ese terreno?”. Don José dijo que no se acordaba y el comisariado ejidal le recomendó que lo buscara. Una semana después de este encuentro, don José

Notas de campo de la audiencia con el comisariado ejidal, agosto de 2014 . 
llegó con un documento que acreditaba la propiedad del terreno.

Después de este acontecimiento, Bertha no sabía qué hacer. Tenía que salirse de la casa, propiedad del suegro. Sin embargo, como pasaba el tiempo y Francisco no le daba la pensión, decidió que era más importante demandar eso y el divorcio, que pelear por la casa, pues no tenía dinero para darle de comer a sus hijos porque no le alcanzaba con la venta de pollos.

$\mathrm{El} \mathrm{CDMCH}$ y Bertha se centraron en interponer tres demandas judiciales: por la violencia que había vivido y denunciado ante la procuradora del DIF municipal, por la pensión alimenticia para sus hijos y por el divorcio. La estrategia del caso, después de hablar con el comisariado ejidal, fue que se reconociera de manera formal la violencia que vivía Bertha con Francisco y el peligro que corrían los hijos al vivir con él. Así podrían pedir la casa a la asamblea ejidal y municipal, con el argumento de la violencia que había vivido. Sin embargo, con el papel de propiedad que tenía, el suegro demandó a Bertha por despojo. Aseguró que había entrado furtivamente a la casa para sacarlo de ahí.

Que Bertha estuviera demandada ante el ministerio público complicaba la estrategia planteada por el CDMCH. Debíamos hacer todo lo que estuviera a nuestro alcance o Bertha perdería su casa, pues para la Ley agraria (Cámara de Diputados, 2017: art. 68 y 69, sección cuarta) el documento de propiedad que don José había entregado era suficiente para quitársela.

También sabíamos que en esta demanda no se tomarían en cuenta las costumbres de la herencia en el matrimonio, los precedentes locales que mostraban que Bertha podía quedarse con su casa por haber sido violentada ni las decisiones de las asambleas de las comunidades. Todas estas cuestiones no valen para la justicia estatal. ¿Cómo podríamos presentar las formas de vida en la comunidad para que tuvieran validez ante el juez que juzgaría a Bertha? La abogada del CDMCH pensó que lo mejor sería elaborar un peritaje antropológico.

\section{El peritaje antropológico como una forma de activismo social para la defensa de los derechos de las mujeres indígenas}

Como se ha argumentado, un peritaje antropológico permite mostrar la diversidad que compone a las sociedades indígenas y evidencia que no todas las realidades pueden ser juzgadas bajo el principio de imparcialidad y neutralidad del derecho. Un informe pericial experto es una ventana a la comprensión de otras realidades y de las desigualdades que se gestan en ellas. Pensamos que el reto principal, al construir este peritaje, era abrir la posibilidad, no sólo de defender a Bertha de las injusticias que enfrentaba, sino también de discutir el derecho de las mujeres a la tierra en su comunidad y la importancia de que las mujeres cuenten con una casa o un pedazo de tierra para trabajar. De esta manera, se visibilizarían las desigualdades de género sobre la tenencia de la tierra. Desde el punto de vista del $\mathrm{CDMCH}$, esto podría detonar un cambio en las costumbres y ayudar a muchas mujeres a obtener precedentes para la tenencia de su tierra.

Estas pretensiones tenían que ver con el objetivo del CDMCH, el cual, además de su trabajo de defensa jurídica, tiene un objetivo político muy claro en la lucha por los derechos de las mujeres de los Altos de Chiapas. Sin embargo, nada se hizo sin la participación de Bertha y de algunos miembros de su comunidad. Intentamos no imponer la investigación sobre el derecho a la decisión de Bertha. Por ello, este peritaje se construyó con la metodología de defensa participativa.

Esta metodología consiste en que las mujeres se involucren en su defensa jurídica y se coloquen como sujetos de derechos ante un sistema jurídico que las victimiza por el simple hecho de ser mujeres indígenas. Con más de cinco años de trabajar con esta metodología, el Centro parte del respeto al pluralismo jurídico de la región de los Altos de Chiapas y busca, en la medida de lo posible, comprender 
las formas de vida propias de los pueblos indígenas, siempre y cuando no violenten los derechos humanos de las mujeres.

En este contexto, el $\mathrm{CDMCH}$ se ha propuesto que las propias mujeres asuman la defensa jurídica de sus casos para que se visualicen como sujetos de derechos y promuevan su autodeterminación. La defensa participativa parte del principio de que:

Una teoría crítica debe pretender efectuar un cambio radical de perspectiva respecto de las teorías tradicionales en la observación del fenómeno jurídico (Facio y Fries, 1999: 19, citado en Olivera, Flores y Padilla, 2015: 103).

Por consiguiente, se determinó que nuestro trabajo debería lograr la participación informada y consciente de las mujeres indígenas y no indígenas, como medio para que asuman de manera directa el ejercicio y defensa de sus derechos, con el acompañamiento de la abogada y las defensoras comunitarias y, a través de esa práctica, fortalecerlas y encaminarlas hacia la transformación de las formas patriarcales de sus culturas. De ahí que a nuestro modelo lo denominemos Defensa Participativa, pues además de proponernos cambiar con él, el posicionamiento de las mujeres ante sus problemas, nos permite revelar directamente al personal de los diferentes sistemas jurídicos en los que actuamos, a través de las mismas actoras, las relaciones de dominación masculina y subordinación femenina que establecen y reproducen durante los procesos, tanto a través de sus discursos, como en el trato que dan a las mujeres y en la parcialidad con que juzgan sus casos (Olivera, Flores y Padi1la, 2015: 108).

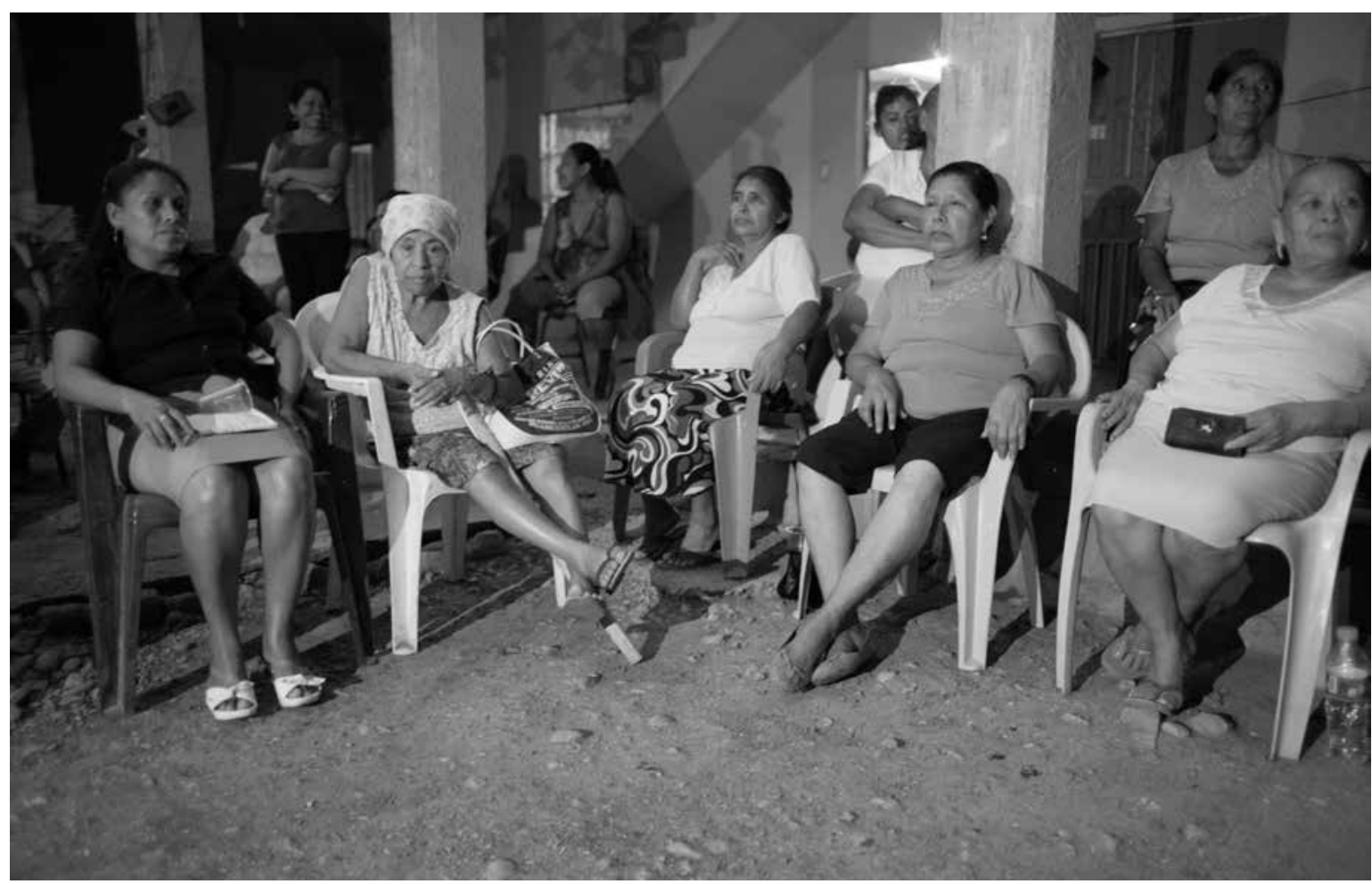

PROMETEO LUCERO Autoridades y pobladores formaron un Concejo Ciudadano. Discuten temas relacionados con la seguridad en Huamuxtitlán, Montaña de Guerrero, febrero de 2013. 
De ahí que en el Centro se afirme, con miras a la integridad que el proceso se propone cumplir, que la defensa participativa, más que ser una defensa jurídica, es una defensa de los derechos de las mujeres.

Ganar el juicio de Bertha significaba, para el Centro, el reconocimiento de los derechos de las mujeres. El fallo a favor ayudaría a legitimar social y culturalmente que la costumbre de heredar la tierra a las mujeres tiene sentido y validez, incluso para el derecho positivo o estatal. En ese sentido, se abonaría de manera positiva a la posibilidad de generar un cambio a favor de las mujeres. Por el contrario, si el fallo fuera en contra, el derecho de las mujeres a la tierra, al menos en la comunidad, quedaría minado y se legitimarían las costumbres que las excluyen. Esta duda ética me acompañó todo el tiempo de la investigación que daría base al peritaje. Aunque sabía que el fallo no dependía sólo de ello, pensaba en las posibilidades que se abrirían o cerrarían. Concluimos que la importancia del peritaje, con independencia de la decisión del juzgado, radicaba en sentar un precedente jurídico que ayudara a la lucha de las mujeres por su derecho a la tierra. Entonces planteamos que el peritaje debía tener por objetivo analizar, con base en la antropología y en la perspectiva de género, la tenencia, uso y usufructo de la tierra en las costumbres de las mujeres casadas en un municipio de los Altos de Chiapas, para demostrar que la demanda contra Bertha tenía su raíz en las desigualdades de poder, que se clarificaban en las desigualdades de género de su comunidad, que afectaban no sólo a la acusada, sino a las mujeres propietarias, y determinar las reparaciones del daño que la demanda le había provocado.

Los ejes de la investigación fueron:

- Vida comunitaria, vida familiar y culturas de género en la comunidad.

- Herencia de la tierra para las mujeres vista desde el género.
- Tenencia, uso y usufructo de la tierra para las mujeres de la comunidad.

- Vida cotidiana y sostenimiento económico de las mujeres de la comunidad.

- Violencia contra las mujeres y su vínculo con la tenencia de la tierra en la comunidad.

- Daños socioeconómicos y culturales vinculados al despojo de la tierra de las mujeres.

Los objetivos y ejes de la investigación se basaban en nuestro acercamiento previo a la comunidad de La Grandeza y a las audiencias anteriores a la demanda que enfrentaba Bertha. Habíamos encontrado algunas pistas que nos permitían fundamentar el peritaje.

Sabíamos que una costumbre en esa comunidad es que los padres heredan al hijo varón un pedazo de tierra, ya sea para vivir o para sembrar, cuando se casa. Esta tierra heredada en el matrimonio no necesita un documento probatorio, pues es una costumbre legítima en la comunidad. La tierra se da porque la práctica del matrimonio supone que será para siempre y que no existen separaciones o divorcios. Entonces la casa heredada a Francisco y Bertha, a los ojos de la costumbre, es de ellos. Por esa razón, nunca se preocuparon por registrarla a su nombre ante las autoridades ejidales. Fundamentar que Francisco era el dueño y no don José hubiera bastado para que fuera legítimo que Bertha se quedara a vivir ahí con sus hijos.

También sabíamos que había antecedentes de violencia en el matrimonio y que los cónyuges se habían separado. En esos casos, las audiencias locales en el juzgado de paz y conciliación indígena habían decidido que la beneficiaria era la mujer porque se quedaba a cargo de los hijos. Las normas y los acuerdos comunitarios decían que las mujeres en estas condiciones se quedarían en las casas y los hombres debían buscar otro lugar donde vivir.

Esto nos ayudaba a fundamentar que Bertha tenía el derecho legítimo de quedarse a vivir en su casa y con el pedazo de tierra en el que se 
encuentran los árboles frutales y verduras que le permitían alimentarse. Al investigar más a profundidad, encontramos que en la comunidad de Bertha la mayoría de las mujeres no son propietarias de sus tierras o casas. La tierra se hereda a los hijos hombres, y cuando las mujeres pueden heredar o tener un pedazo de tierra para vivir o sembrar, se enfrentan a problemas con la asamblea ejidal porque no tienen derecho a participar en las decisiones de la comunidad y a decidir sobre sus tierras igual que los hombres, y tampoco pueden acceder a apoyos gubernamentales para el campo. Las pocas mujeres propietarias deben hacer frente a las críticas de la comunidad y al desprestigio porque al reclamar la tierra están rompiendo las costumbres sobre la tenencia y el orden de género establecido, lo que provoca violencias contra ellas.

Todas estas pistas nos mostraban los cambios y las continuidades acerca del derecho de las mujeres a la tenencia de la tierra. Veíamos, por un lado, que ciertas costumbres estaban siendo cuestionadas por las propietarias, y por el otro, las resistencias de la comunidad a la independencia que la tierra podía dar a las mujeres.

Por medio de la información recabada en las entrevistas con los familiares de Bertha, en los grupos focales de las mujeres organizadas y en las entrevistas y grupos focales con las autoridades de la comunidad de La Grandeza, descubrimos que detrás de la decisión de don José de quitarle la casa a Bertha estaba la costumbre de que las mujeres no podían tener acceso a la propiedad. Dejarle la casa y la tierra no sólo significaba que Bertha tomara las riendas de su vida, sino perder una propiedad. Como lo había mencionado en la audiencia con el comisariado ejidal, si Bertha encontraba otra pareja, la propiedad pasaría en automático al hombre del hogar, pues por años ellos han mediado la vida de las mujeres en el ámbito público.

Para no seguir reproduciendo la visión errónea de que las costumbres de las comunidades indígenas son "machistas y violentas" con las mujeres, decidimos plasmar en el peritaje la continuidad de las costumbres de la herencia en el matrimonio y la propiedad de las casas cuando se vive violencia. Era importante hacer ver que existían cambios y continuidades en la propiedad de la tierra de las mujeres, que se enmarcaban en el orden de género establecido y podían llevar o no a la desigualdad entre mujeres y hombres, y aunque lograran su derecho a la tierra, las mujeres quedaban en mayor vulnerabilidad.

Buscamos contextualizar las costumbres y mostrar que en algunas subyacen opresiones enraizadas en los sistemas comunitarios. De ahí que en la construcción del peritaje siempre tuvimos presentes las preguntas sobre las implicaciones éticas de afirmar o deslegitimar ciertas costumbres y cuál sería el impacto en las dinámicas de las comunidades. Era necesario señalar que las costumbres y tradiciones de los pueblos indígenas no son inamovibles y estáticas, por el contrario, son prácticas vivas y dinámicas, que muestran que muchas mujeres de la comunidad de La Grandeza pueden contar con un pedazo de tierra para sembrar o vivir. Por lo tanto, que Bertha quisiera quedarse con la casa no interfería con la forma de vida de su comunidad, sino que es un derecho al que podía acceder. El impacto de lo anterior no sólo tenía que ver con hacer visible la injusticia contra Bertha, sino con la lucha del Centro a favor del derecho de las mujeres indígenas a la tenencia de la tierra con el objetivo de la defensa participativa, que se centra en la defensa jurídica y en hacer explícitos los derechos de las mujeres.

Para lograr que el peritaje antropológico sirviera también como un instrumento de denuncia de los derechos de las mujeres y no sólo para defender a Bertha, necesité tener una posición diferente como perita antropóloga y como feminista. Las abogadas del Centro se despojaron también de sus supuestos de imparcialidad del derecho y combinaron sus saberes jurídicos con su militancia política. Tomar esta decisión con Bertha nos llevó a construir 
acciones y una investigación más comprometidas con la realidad social, en la que los pueblos indígenas se encuentran en desventaja sustancial y las mujeres indígenas buscan defender sus derechos.

En este sentido, el informe pericial apuntó a ser un aporte más de lo que Hernández (2017) llama una investigación activista a partir de la antropología jurídica feminista, corriente que se ha consolidado en experiencias de investigación hechas con base en la intersección del activismo y la investigación académica. Hacer investigación desde una perspectiva activista y colaborativa implica nuevos "dilemas éticos y epistemológicos" para la antropología - en este caso, para la construcción de peritajes culturales con perspectiva de género-, ya que elaborar un peritaje cultural "reproduce las jerarquías en torno al conocimiento, legitimando el conocimiento cultural del antropólogo por sobre el de los mismos pueblos indígenas” (2017: 16). Desde una visión crítica, Hernández argumenta que parece que sólo "los antropólogos [somos] los que tenemos el conocimiento cultural legítimo que puede ser reconocido por los operadores de justicia, y en ese sentido tenemos la última palabra en torno a lo que es la 'verdadera práctica cultural indígena' o el 'verdadero derecho indígena”" (2017: 16).

Esta situación ya es cuestionada por académicos indígenas activistas, que han criticado fuertemente las lógicas tradicionalistas en la elaboración de peritajes culturales. Algunos jueces prefieren llamar a antropólogos mestizos para "traducir" la vida comunitaria, lo que sigue una lógica racista que reproduce la visión de que las personas indígenas no tienen la capacidad de construir un peritaje de su propia cultura, al mismo tiempo que atrapa a los antropólogos entre la perspectiva estatal y su posición al margen del Estado.

En la actualidad, gracias a estas críticas, las organizaciones de la sociedad civil han impulsado el uso de peritajes culturales con perspectiva de género y han comenzado a abrir espacios para académicos activistas indígenas que han construido o participado en la elaboración de estos informes. Esto nos exige, aún más, abrirnos a los cambios que la disciplina nos impone. Hernández (2017) plantea que, ante estos retos, es necesario construir formas más participativas y dialógicas que contribuyan a reflexionar de manera colectiva y a cuestionar los discursos de poder evidentes en las prácticas del derecho.

Esperamos que este aporte de investigación participativa sea un ejemplo de cómo llevar a cabo investigaciones comprometidas, que sirvan a los fines de los sectores o personas más afectadas. Es importante decir que, después de cuatro años, el caso de Bertha sigue abierto en un ministerio público. Mientras el caso no se retome, ella sigue viviendo en su casa con sus hijos. También se encuentra a la espera de una resolución de sus demandas de pensión alimenticia y divorcio, que lejos de debilitarla, la han fortalecido.

\section{Conclusiones}

En este artículo se analizó la experiencia de construcción de un peritaje cultural como una forma de colaboración investigativa con el $\mathrm{CDMCH}$ para el caso de Bertha, una mujer tseltal que reclamó su derecho a la propiedad y la tierra, y desafió el orden de género en su comunidad.

Escribir un informe pericial cultural con perspectiva de género pone en el centro del debate la imparcialidad del derecho, pues se hacen visibles las desigualdades de género en el sistema jurídico y la discriminación hacia las mujeres indígenas en el acceso a sus derechos.

A lo largo de este análisis se desvelaron los problemas enmarcados en la aplicación del derecho y el acceso a la justicia, que muestran que el campo del derecho está en disputa constante, en el que se clarifica el orden de género establecido. En este sentido, la experiencia analizada nos permite describir 
el dinamismo en el campo de derecho, pues está en constante cambio y adaptación.

Este dinamismo ha permitido que el peritaje antropológico sirva no sólo como una prueba para desvelar la cultura de los pueblos indígenas, sino que puede ser utilizado por la lucha política de las comunidades, impulsada por las organizaciones de la sociedad civil. Esto implica nuevos retos para las ciencias antropológica y jurídica, que permitirán caminar hacia la pluralidad, el acceso de las mujeres a la justicia y a la construcción de una sociedad más equitativa y justa. D

Bibliografía

Cámara de Diputados, 2017, Ley agraria. Disponible en línea: <http://www.diputados.gob.mx/LeyesBiblio/ref/lagra.htm>.

Comisión Nacional para el Desarrollo de los Pueblos Indígenas (CDI), 2003, Convenio 169 de la olt sobre Pueblos Indígenas y Tribales en Países Independientes, Comisión Nacional para el Desarrollo de los Pueblos Indígenas (Cuadernos de legislación indígena), México.

Congreso de la Unión, 1991, "Decreto por el que se modifican diversas disposiciones del Código Federal de Procedimientos Penales y del Código de Procedimientos Penales para el Distrito Federal”, en Diario Oficial de la Federación, 8 de enero. Disponible en línea: <http:// www.dof.gob.mx/nota_detalle.php?codigo=4698368\&fecha=08/01/1991>

__ 2007, "Decreto por el que se expide la Ley General de Acceso de las Mujeres a una Vida Libre de Violencia", en Diario Oficial de la Federación, 1 de febrero. Disponible en línea: <http://www.dof.gob.mx/nota_detalle.php?codigo=4961209\&fecha=01/02/2007>.

Crenshaw, Kimberlé, 1995, "Mapping the Margins: Intersectionality, Identity Politics and Violence against Women of Color", en Kimberlé Crenshaw, Neil T. Gotanda, Gary Peller y Kendall Thomas (eds.), Critical Race Theory, New Press, Nueva York, pp. 357-383.

Escalante Betancourt, Yuri, 2002, La experiencia del peritaje antropológico, Instituto Nacional Indigenista/Secretaría de Desarrollo Social, México.

Estrada Mendoza, María de la Luz (coord.), 2016, Guía metodológica para la construcción de peritajes antropológicos, psicosociales y socioculturales en casos de feminicidio en México, Católicas por el Derecho a Decidir/Observatorio Ciudadano Nacional del Feminicidio/ Fondo de Canadá, México.

Facio, Alda y Lorena Fries, 1999, “Feminismo, género y patriarcado", en Alda Facio y Lorena Fries (eds.), Género y derecho, LOM, Santiago de Chile, pp. 6-42.

Haraway, Donna, 1995, “Conocimientos situados: la cuestión científica en el feminismo y el privilegio de la perspectiva parcial”, en Donna Haraway, Ciencia, cyborgs y mujeres. La reinvención de la naturaleza, Cátedra, Madrid, pp. 313-346.

Hernández Castillo, Rosalva Aída, 2017, “Activismo legal y las paradojas de la antropología jurídica feminista”, en Rosalva Aída Hernández Castillo (coord.), Resistencias penitenciarias: investigación activista en espacios de reclusión, Grupo Internacional de Trabajo sobre Asuntos Indígenas/Colectiva Editorial Hermanas en la Sombra/Libera Desarrollo Humano/Juan Pablos, México, pp. 51-82.

Martínez, Juan Carlos y Daniela Ramírez, 2012, “Caso Irene Cruz Zúñiga: Informe pericial antropológico con perspectiva de género”, documento de trabajo.

Presidencia de la República, 1981, "Decreto de Promulgación de la Convención Americana sobre Derechos Humanos, adoptada en la ciudad de San José de Costa Rica, el 22 de noviembre de 1969", en Diario Oficial de la Federación, 7 de mayo. Disponible en línea: <http://www. dof.gob.mx/nota_detalle.php?codigo=4645612\&fecha=07/05/1981>.

Olivera, Mercedes, Gloria Flores y Alma Padilla, 2015, “Metodología de la defensa participativa”, en María Estela Lanari y Claudia Hasanbegovic (comps.), Mujeres de Latinoamérica, Editorial de la Universidad Nacional de Mar del Plata, Mar del Plata, pp. 101-128.

Ortiz, Héctor, 2000, La diferencia cultural en el ámbito legal: el trabajo pericial antropológico, tesis de licenciatura en etnología, Escuela Nacional de Antropología e Historia, México.

Saavedra Hernández, Laura Edith, 2018, "La experiencia en la metodología colaborativa a través de la construcción de un peritaje antropológico con perspectiva de género: el caso de Alberta", en Laura Edith Saavedra Hernández, Construyendo justicia(s) más allá de la ley: las experiencias de las mujeres indígenas que participan con el Centro de Derechos de la Mujer de Chiapas, A. C., tesis de doctorado en antropología, Centro de Investigaciones y Estudios Superiores en Antropología Social, México, pp. 219-249. 
Sánchez Botero, Esther, 2010, El peritaje antropológico. Justicia en clave cultural, Deutsche Gesellschaft für Technische Zusammenarbeit/ Cooperación Técnica Alemana/ProFis, Bogotá.

Secretaría de Relaciones Exteriores (SRE), 2008, Convención Interamericana para Prevenir, Sancionar y Erradicar la Violencia contra la Mujer, "Convención de Belém do Pará" y su Estatuto de Mecanismo y Seguimiento, Secretaría de Relaciones Exteriores/Fondo de Desarrollo de las Naciones Unidas para la Mujer/Programa de las Naciones Unidas para el Desarrollo, México.

Valladares, Laura, 2012, "La importancia del peritaje cultural: avances, retos y acciones del Colegio de Etnólogos y Antropólogos Sociales (CEAS) para la certificación de peritos”, en Boletín del Colegio de Etnólogos y Antropólogos Sociales, año 2012, pp. 11-20. 